\title{
A logarithmic Gauss curvature flow and the Minkowski problem
}

by

\author{
Kai-Seng CHOU ${ }^{\mathrm{a}, 1}$, Xu-Jia WANG ${ }^{\mathrm{b}, 2}$ \\ a Department of Mathematic, The Chinese University of Hong Kong, \\ Shatin, Hong Kong \\ b School of Mathematical Sciences, Australian National University, \\ Canberra, ACT0200, Australia
}

Manuscript received 18 October 1999, revised 25 April 2000

ABSTRACT. - Let $X_{0}$ be a smooth uniformly convex hypersurface and $f$ a postive smooth function in $S^{n}$. We study the motion of convex hypersurfaces $X(\cdot, t)$ with initial $X(\cdot, 0)=\theta X_{0}$ along its inner normal at a rate equal to $\log (K / f)$ where $K$ is the Gauss curvature of $X(\cdot, t)$. We show that the hypersurfaces remain smooth and uniformly convex, and there exists $\theta^{*}>0$ such that if $\theta<\theta^{*}$, they shrink to a point in finite time and, if $\theta>\theta^{*}$, they expand to an asymptotic sphere. Finally, when $\theta=\theta^{*}$, they converge to a convex hypersurface of which Gauss curvature is given explicitly by a function depending on $f(x)$.

(C) 2000 L'Association Publications de l'Institut Henri Poincaré. Published by Elsevier B.V. All rights reserved

Key words: Parabolic Monge-Ampère equation, Gauss curvature, Minkowski problem, Asymptotic behavior

AMS classification: $35 \mathrm{~K} 15,35 \mathrm{~K} 55,58 \mathrm{G}$

\footnotetext{
${ }^{1}$ E-mail: kschou@ @math.cuhk.edu.hk.

${ }^{2}$ E-mail: wang@wintermute.anu.edu.au.
} 


\section{INTRODUCTION}

Let $f$ be a positive smooth function defined in the $n$-dimensional sphere $S^{n}$ and let $X_{0}: S^{n} \rightarrow \mathbf{R}^{n+1}$ be a parametrization of a smooth, uniformly convex hypersurface $M_{0}$. In this paper we are concerned with the motion of the convex hypersurfaces $M(t)$ satisfying the equation

$$
\frac{\partial X}{\partial t}=-\log \frac{K(v)}{f(v)} v,
$$

with $X(p, 0)=X_{0}(p)$. Here for each $t X(\cdot, t)$ parametrizes $M(t)$, $K(v(p, t))$ is the Gauss curvature of $M(t)$ and $v(p, t)$ is the unit outer normal at $X(p, t)$. Notice that by strict convexity the Gauss curvature can be regarded as a function of the normal. Recall that a uniformly convex hypersurface is a hypersurface with positive Gaussian curvature and hence it is stricly convex.

Our study on (0.1) is motivated by the search for a variational proof of the classical Minkowski problem in the smooth category. Recall that for a convex hypersurface the inverse of its Gauss map induces a Borel measure on the unit sphere called the area measure of the hypersurface. Naturally one asks when a given Borel measure on $S^{n}$ is the area measure of some convex hypersurface. This problem was formulated and solved by Minkowski [13] for polytopes in 1897 by a variational argument. Later he extended his result to cover all Borel measures which are of the form $1 / f d \sigma$ where $f$ is continuous and $d \sigma$ is the standard Lebsegue measure on $S^{n}$ [14]. The regularity of the convex hypersurface realizing the area measure was not considered by Minkowski. Thus it led to the Minkowski problem in the smooth category, namely, when is a positive, smooth function in $S^{n}$ the Gauss curvature of a smooth convex hypersurface? There are two approaches for this problem. On one hand, the method of continuity was used by Lewy [12], Miranda [15], Nirenberg [16], and Cheng and Yau [3]. On the other hand, a regularity theory was developed for the generalized solution (see Pogorelov [17]).

Let $M$ be a convex hypersurface and $V(M)$ its enclosed volume. We have

$$
V(M)=\frac{1}{n+1} \int_{S^{n}} \frac{H(x)}{K(x)} d \sigma(x),
$$

where $H$ and $K$ are respectively the support function and Gauss curvature of $M$. When expressed in the smooth category, Minkowski's 
original proof is to show that the solution is the convex hypersurface which minimizes the functional $\int H(x) / f(x) d \sigma(x)$ over all convex hypersurfaces of the same enclosed volume. In view of this we may consider the functional

$$
J(M)=-V(M)+\int_{S^{n}} \frac{H}{f} d \sigma .
$$

It is not hard to see that $(0.1)$ is a negative gradient flow for $J$. By a careful study of this flow, we shall give another proof of the Minkowski problem in the smooth category.

THEOREM A. - Let $X_{0}$ be a smooth uniformly convex hypersurface. For $\theta>0$, consider $(0.1)$ subject to

$$
X(\cdot, 0)=\theta X_{0} .
$$

There exists $\theta^{*}>0$ such that the flow $X(\cdot, t)$ beginning at $\theta^{*} X_{0}$ tends to a smooth uniformly convex hypersurface $X^{*}$ in the sense that

$$
X(\cdot, t)-\xi t \rightarrow X^{*},
$$

smoothly as $t \rightarrow \infty$ where $\xi$ is uniquely determined by

$$
\int_{S^{n}} \frac{x_{i}}{\mathrm{e}^{\xi \cdot x} f(x)} d \sigma(x)=0, \quad i=1, \ldots, n+1 .
$$

Furthermore, the Gauss curvature of $X^{*}$, when regarded as a function of the normal, is equal to $\mathrm{e}^{\xi \cdot x} f(x)$.

THEOREM B. - Let $\theta^{*}$ be as in Theorem A. If $\theta \in\left(0, \theta^{*}\right)$, the solution of $(0.1),(0.2)$ shrinks to a point in finite time. If $\theta \in\left(\theta^{*}, \infty\right)$, the solution expands to infinity as $t$ goes to infinity. In the latter case, the hypersurface $X(\cdot, t) / r(t)$ where $r(t)$ is the inner radius of $X(\cdot, t)$ converges to a unit sphere uniformly.

As a direct consequence of Theorem A we have

COROLlaRY (Minkowski problem). - A positive, smooth function $f$ in $S^{n}$ is the Gauss curvature of a uniformly convex hypersurface if and only if it satisfies

$$
\int_{S^{n}} \frac{x_{i}}{f(x)} d \sigma(x)=0, \quad i=1, \ldots, n+1 .
$$


Theorems A and B will be proved in the following sections by an approach similar to that used in [4], namely, by introducing the support function of $X(\cdot, t)$ and reducing $(0.1)$ to a single parabolic equation of Monge-Ampère type for its support function. In Section 1 we collect some facts on the support function of a convex hypersurface. In Section 2 a priori estimates for the support function, in particular upper and lower bounds for the second derivatives, will be derived. They are used in Section 3 to establish Theorems A and B.

Motion of convex hypersurfaces driven by functions of Gauss curvature of the form

$$
\frac{\partial X}{\partial t}=\Phi(v, K) v
$$

has been studied by several authors including Andrews [1], Chou [4], Chow [7], Frey [8], Gerhardt [10] and Urbas [18]. When $\Phi=-K^{\sigma}$, $\sigma>0$, it was proved in [7] that $M(t)$ exists and shrinks to a point in finite time. Moreover, it becomes asymptotically round when $\sigma$ is equal to $1 / n$. In [1] it was shown that $M(t)$ becomes an asymptotic ellipsoid when $\sigma$ is equal to $1 /(n+2)$. Expanding flows rather than contracting ones were studied in [10] and [18]. For a class of curvature functions including $\Phi=K^{-1 / n}$ it was proved that $M(t)$ expands to infinity like a sphere in infinite time. In all these results $\Phi$ is independent of $v$. For anisotropic flows very little is known. We mention the works Andrew [2], Chou and Zhu [6], and Gage and Li [9].

\section{THE SUPPORT FUNCTION}

In this section we collect some basic facts concerning a convex hypersurface and its support function. Details can be found in Cheng and Yau [3] and Pogorelov [17].

Let $M$ be a closed convex hypersurface in $\mathbf{R}^{n+1}$. Its support function $H$ is defined on $S^{n}$ by

$$
H(x)=\sup \{x \cdot p: p \in M\},
$$

where $x \cdot p$ is the inner product in $\mathbf{R}^{n+1}$. We extend $H$ to a homogenuous function of degree 1 in $\mathbf{R}^{n+1}$. So $H$ is convex and satisfies

$$
\sup _{S^{n}}|\nabla H| \leqslant \sup _{S^{n}}|H|,
$$


since it is the supremum of linear functions. If $M$ is strictly convex, that is, for each $x$ in $S^{n}$ there is a unique point $p$ on $M$ whose unit outer normal is $x, H$ is differentiable at $x$ and

$$
p_{i}=\frac{\partial H}{\partial x_{i}}, \quad i=1, \ldots, n+1 .
$$

Thus the map $x \mapsto p(x)$ gives a parametrization of $M$ by its normal. In fact, it is nothing but the inverse of the Gauss map.

Geometric quantities of $M$ can now be expressed through $H$. Let $e_{1}, \ldots, e_{n}$ be an orthonormal frame fields on $S^{n}$. By a direct computation one sees that the principal radii of curvature at $p(x)$ are precisely the eigenvalues of the matrix $\left(\nabla_{\beta} \nabla_{\alpha} H+H \delta_{\alpha \beta}\right)_{, \alpha, \beta=1, \ldots, n}$, where $\nabla_{\alpha}$ is the covariant differentiation with respect to $e_{\alpha}$. In particular, the Gauss curvature at $p(x)$ is given by

$$
K(x)=1 / \operatorname{det}\left(\nabla_{\beta} \nabla_{\alpha} H+H \delta_{\alpha \beta}\right) .
$$

When $H$ is viewed as a homogeneous function over $\mathbf{R}^{n+1}$, the principal radii of curvature of $M$ are also equal to the non-zero eigenvalues of the Hessian matrix $\left(\partial^{2} H / \partial x_{i} \partial x_{j}\right)_{i, j=1, \ldots, n+1}$.

Now we can reduce the problem $(0.1),(0.2)$ to an initial value problem for the support function. In fact, let $H(x, t)$ be the support function of $M(t)$. By definition we have

$$
x \cdot \frac{\partial X}{\partial t}(p(x), t)=-\frac{\partial H}{\partial t}(x, t) .
$$

From (0.1) and (0.2) it follows that $H$ satisfies

$$
\begin{aligned}
\frac{\partial H}{\partial t} & =\log \operatorname{det}\left(\nabla_{\beta} \nabla_{\alpha} H+H \delta_{\alpha \beta}\right) f, \\
H(x, 0) & =\theta H_{0}(x),
\end{aligned}
$$

where $H_{0}$ is the support function for $M_{0}$. Conversely, if $X(\cdot, t)$ is a family of convex hypersurfaces determined by a solution of (1.3) and (1.4), it is not hard to see that $X(\cdot, t)$ does solve $(0.1)$ and $(0.2)$. See, for instance, [4] for details. Notice from (1.3) $H(x, t)$ must determine a uniformly convex hypersurface.

Eq. (1.3) has a variational structure. Consider the enclosed volume of a uniformly convex hypersurface $M$, 


$$
\begin{aligned}
V(M) & =\frac{1}{n+1} \int_{S^{n}} \frac{H(x)}{K(x)} d \sigma(x) \\
& =\frac{1}{n+1} \int_{S^{n}} H \operatorname{det}\left(\nabla_{\beta} \nabla_{\alpha} H+H \delta_{\alpha \beta}\right) d \sigma .
\end{aligned}
$$

Regarding $V$ as a functional on support functions, we find that the first variation of $V$ is

$$
\delta V(H) h=\int_{S^{n}} h \operatorname{det}\left(\nabla_{\beta} \nabla_{\alpha} H+H \delta_{\alpha \beta}\right) d \sigma,
$$

where $h$ is any smooth function. Let's consider the functional $J$ defined on all uniformly convex hypersurfaces

$$
J(H)=-V(H)+\int_{S^{n}} \frac{H}{f} d \sigma,
$$

where $f$ is positive. When $H$ is a solution of (1.3),

$$
\begin{aligned}
\frac{d}{d t} J(H(\cdot, t)) & =-\int_{S^{n}}\left[\operatorname{det}\left(\nabla_{\beta} \nabla_{\alpha} H+H \delta_{\alpha \beta}\right)-\frac{1}{f}\right] \frac{\partial H}{\partial t} d \sigma \\
& =-\int_{S^{n}} \frac{1}{f}\left(\mathrm{e}^{H_{t}}-1\right) H_{t} d \sigma \\
& \leqslant 0 .
\end{aligned}
$$

Hence (1.3) is a negative gradient flow for $J$. (1.5) will be used in the proof of Theorem A. This variational approach to the problem of prescribed Gauss curvature was first adopted in Chou [5].

To obtain apriori estimates for the higher derivatives for $H$ it is convenient to express Eq. (1.3) locally in the Euclidean space. Thus let $u(y, t)$ be the restriction of $H(x, t)$ to the hypersurface $x_{n+1}=-1$, i.e., $u(y, t)=H(y,-1, t)$. Then $u$ is convex in $\mathbf{R}^{n}$ and we have

$$
\operatorname{det} \nabla^{2} u(y, t)=\left(1+|y|^{2}\right)^{-\frac{n+2}{2}} \operatorname{det}\left(\nabla_{\beta} \nabla_{\alpha} H+H \delta_{\alpha \beta}\right)(x, t)
$$

and

$$
\frac{\partial u}{\partial t}(y, t)=\sqrt{1+|y|^{2}} \frac{\partial H}{\partial t}(x, t)
$$


for $x=(y,-1) / \sqrt{1+|y|^{2}}$. Extend $f$ to be a homogenuous function of degree 0 in $\mathbf{R}^{n+1}$. We get

$$
\frac{\partial u}{\partial t}=\sqrt{1+|y|^{2}} \log \operatorname{det} \nabla^{2} u+g(y), \quad y \in \mathbf{R}^{n},
$$

where

$$
g(y)=\sqrt{1+|y|^{2}}\left[\frac{n+2}{2} \log \left(1+|y|^{2}\right)+\log f(y,-1)\right] .
$$

\section{A PRIORI ESTIMATION}

First of all we note that the uniqueness of solution to (1.3), (1.4) follows from the following comparison principle which is a direct consequence of the maximum principle.

LEMMA 2.1. - For $i=1,2$, let $f_{i}$ be two positive $C^{2}$-functions on $S^{n}$ and $H_{i} C^{2,1}$-solutions of

$$
\frac{\partial H}{\partial t}=\log \operatorname{det}\left(\nabla_{\beta} \nabla_{\alpha}+H \delta_{\alpha \beta}\right) f_{i} .
$$

Suppose that $H_{1}(x, 0) \leqslant H_{2}(x, 0)$ and $f_{1}(x) \leqslant f_{2}(x)$ on $S^{n}$. Then $H_{1} \leqslant$ $H_{2}$ for all $t>0$ and $H_{1}<H_{2}$ unless $H_{1} \equiv H_{2}$.

In the following we shall always assume $H \in C^{4,2}\left(S^{n} \times[0, T]\right)$ is a solution of (1.3), (1.4). Let $R(t)$ and $r(t)$ be the outer and inner radii of the hypersurface $X(\cdot, t)$ determined by $H(x, t)$ respectively. We set

$$
R_{0}=\sup \{R(t): t \in[0, T]\}
$$

and

$$
r_{0}=\inf \{r(t): t \in[0, T]\} .
$$

We shall estimate the principal radii of curvatures of $X(\cdot, t)$ from both side in terms of $r_{0}^{-1}, R_{0}$, and initial data.

LEMMA 2.2. - Let $r$ and $R$ be the inner and outer radii of a uniformly convex hypersurface $X$ respectively. Then there exists a dimensional constant $C$ such that

$$
\frac{R^{2}}{r} \leqslant C \sup \left\{R(x, \xi): x, \xi \in S^{n}\right\},
$$


where $R(x, \xi)$ is the principal radius of curvature of $X$ at the point with normal $x$ and along the direction $\xi$.

Proof. - For any given $t>0$, let

$$
h=\inf \left\{H(x)+H(-x): x \in S^{n}\right\} .
$$

Then $X$ is pinched between two parallel hyperplanes with distance $h$. Suppose the infimum is attained at $x=(1,0, \ldots, 0)$. By convexity we can choose a direction perpendicular to the $x_{1}$-axis, say, the $x_{2}$-axis such that

$$
H(0,1,0, \ldots, 0)+H(0,-1,0, \ldots, 0) \geqslant \frac{1}{2} R .
$$

Let $F$ be the projection of $X$ on the plane $x_{3}=\cdots=x_{n+1}=0$. Then $F$ is a convex set and its diameter is larger than $\frac{1}{2} R$. By a proper choice of the origin we may assume $F$ is contained in $\left\{-h<x_{1}<h\right\}$ and $\left\{0, \pm \frac{1}{8} R\right\}$ belongs to $F$. By projection we see that the supremum of the principal radii of curvatures of the boundary of $F$ cannot exceed that of $X$.

Let $E$ be the ellipse given by

$$
\frac{x_{1}^{2}}{b^{2}}+\frac{x_{2}^{2}}{(R / 16)^{2}}=1
$$

where $b$ is chosen so that $E \subset F$ and $\partial E \cap \partial F$ is non-empty. Then $h / 4 \leqslant b \leqslant h / 2$ provided $R \gg r$. For any $\left(\bar{x}_{1}, \bar{x}_{2}\right) \in \partial E \cap \partial F$, since $\left(0, \pm \frac{1}{8} R\right) \in F$, we have $\left|\bar{x}_{1}\right| \geqslant b / 2$. Hence $\left|\bar{x}_{2}\right| \leqslant \sqrt{3} R / 32$. Simple computation shows that the principal radius of curvature of the boundary of $F$ at $\left(\bar{x}_{1}, \bar{x}_{2}\right)$ is larger than $R^{2} / 8^{3} b$. Hence by noticing $b \leqslant r$ we obtain

$$
\frac{R^{2}}{r} \leqslant C \frac{R^{2}}{b} \leqslant C \sup _{x, \xi} R(x, \xi) .
$$

LEMMA 2.3. - Suppose that $a(t), b(t) \in C^{1}([0, T])$ and $a(t)<b(t)$ for all $t$. Then there exists $h(t) \in C^{0,1}([0, T])$ such that

(1) $a(t)-2 M \leqslant h(t) \leqslant b(t)+2 M$;

(2) $\sup \left\{\frac{\left|h\left(t_{1}\right)-h\left(t_{2}\right)\right|}{\left|t_{1}-t_{2}\right|}: t_{1}, t_{2} \in[0, T]\right\} \leqslant 2 \max \left\{\sup _{t} b^{\prime}(t), \sup _{t}\left(-a^{\prime}(t)\right)\right\}$, where $M=\sup _{t}(b(t)-a(t))$.

Proof. - We define $h(t)$ step by step. Let $t_{0}=0$, and $h_{0}=(a(0)+$ $b(0)) / 2$. For $j \geqslant 1$, let 


$$
\begin{gathered}
t_{j}=\sup \left\{\tau \in\left(t_{j-1}, T\right):\right. \\
\quad a(t) \geqslant h_{j-1}-M, b(t) \leqslant h_{j-1}+M, \\
\left.\forall t \in\left(t_{j-1}, \tau\right)\right\}, \\
h_{j}=\frac{1}{2}\left(a\left(t_{j}\right)+b\left(t_{j}\right)\right),
\end{gathered}
$$

and

$$
h(t)=h_{j-1}+\frac{h_{j}-h_{j-1}}{t_{j}-t_{j-1}}\left(t-t_{j-1}\right) \quad \text { for } t \in\left(t_{j-1}, t_{j}\right) .
$$

Then $h(t)$ is the desired function.

Now we give an upper estimate for the principal radii of curvature.

LEMMA 2.4. - For any $\gamma \in(1,2]$ there exists a constant $C_{\gamma}$, which may depend on initial data, such that

$$
\sup \left\{H_{\xi \xi}(x, t): \xi \text { tangential to } S^{n}\right\} \leqslant C_{\gamma}\left(1+D^{\gamma}\right),
$$

where $D=\sup \{d(t): t \in[0, T]\}$ and $d(t)$ is the diameter of $X(\cdot, t)$.

Proof.-Applying Lemma 2.3 to the functions $-H\left(-e_{i}, t\right)$ and $H\left(e_{i}, t\right)$ where $\pm e_{i}$ are the intersection points of $S^{n}$ with the $x_{i}$-axis, $i=1, \ldots, n+1$, we obtain $p_{i}(t)$ so that

$$
-H\left(-e_{i}, t\right)-2 D \leqslant p_{i}(t) \leqslant H\left(e_{i}, t\right)+2 D
$$

and

$$
\begin{aligned}
& \sup \left\{\frac{\left|p_{i}\left(t_{1}\right)-p_{i}\left(t_{2}\right)\right|}{\left|t_{1}-t_{2}\right|}: t_{1}, t_{2} \in[0, T]\right\} \\
& \quad \leqslant 2 \sup \left\{H_{t}(x, t):(x, t) \in S^{n} \times[0, T]\right\} .
\end{aligned}
$$

Henceforth

$$
\left|H(x, t)-\sum_{i=1}^{n+1} p_{i}(t) x_{i}\right| \leqslant 2 D \quad \text { for }(x, t) \in S^{n} \times[0, T],
$$

and by (1.1)

$$
\sum_{i=1}^{n+1}\left|H_{i}(x, t)-p_{i}\right|^{2} \leqslant 4 D^{2}
$$


Let

$$
\Phi(x, t)=H_{\xi \xi}(x, t)+\left[1+\sum_{i=1}^{n+1}\left|H_{i}(x, t)-p_{i}(t)\right|^{2}\right]^{\gamma / 2}
$$

where $\gamma \in(1,2]$. Suppose that the supremum

$$
\sup \left\{\Phi(x, t):(x, t) \in S^{n} \times[0, T], \xi \text { tangential to } S^{n},|\xi|=1\right\}
$$

is attained at the south pole $x=(0, \ldots, 0,-1)$ at $t=\bar{t}>0$ and in the direction $\xi=e_{1}$. For any $x$ on the south hemisphere, let

$$
\xi(x)=\left(\sqrt{1-x_{1}^{2}},-\frac{x_{1} x_{2}}{\sqrt{1-x_{1}^{2}}}, \ldots,-\frac{x_{1} x_{n+1}}{\sqrt{1-x_{1}^{2}}}\right) .
$$

Let $u$ be the restriction of $H$ on $x_{n+1}=-1$. Using the homogenity of $H$ we obtain, after a direct computation,

$$
\begin{aligned}
& \sum_{i=1}^{n+1}\left(H_{i}-p_{i}\right)^{2}(x, t) \\
& \quad=\sum_{i=1}^{n}\left(u_{i}(y, t)-p_{i}(t)\right)^{2}+\left|u(y, t)+p_{n+1}-\sum_{i=1}^{n} y_{i} u_{i}(y, t)\right|^{2}
\end{aligned}
$$

and

$$
H_{\xi \xi}(x, t)=u_{11}(y, t) \frac{\left(1+y_{1}^{2}+\cdots+y_{n}^{2}\right)^{3 / 2}}{1+y_{2}^{2}+\cdots+y_{n}^{2}},
$$

where $y=-\left(x_{1}, \ldots, x_{n}\right) / x_{n+1}$ in $\mathbf{R}^{n}$. Thus the function

$$
\begin{aligned}
\varphi(y, t)= & u_{11} \frac{\left(1+y_{1}^{2}+\cdots+y_{n}^{2}\right)^{3 / 2}}{1+y_{2}^{2}+\cdots+y_{n}^{2}} \\
& +\left[1+\sum\left(u_{i}-p_{i}\right)^{2}+\left|u+p_{n+1}-\sum y_{i} u_{i}\right|^{2}\right]^{\gamma / 2}
\end{aligned}
$$

attains its maximum at $(y, t)=(0, \bar{t})$. Without loss of generality we may further assume that the Hessian of $u$ at $(0, \bar{t})$ is diagonal. Hence at $(0, \bar{t})$ we have, for each $k$,

$$
\begin{aligned}
0 \leqslant \varphi_{t}= & u_{11 t}+\gamma\left[\left(u_{i}-p_{i}\right)\left(u_{i t}-p_{i, t}\right)\right. \\
& \left.+\left(u+p_{n+1}\right)\left(u_{t}+p_{n+1, t}\right)\right] Q^{(\gamma-2) / 2}, \\
0=\varphi_{k}= & u_{11 k}+\gamma\left(u_{i}-p_{i}\right) u_{i k} Q^{(\gamma-2) / 2},
\end{aligned}
$$


and

$$
\begin{aligned}
0 \geqslant \varphi_{k k}= & u_{k k 11}+\tau_{k} u_{11}+\gamma\left[u_{k k}^{2}+\left(u_{i}-p_{i}\right) u_{i k k}\right. \\
& \left.-\left(u+p_{n+1}\right) u_{k k}\right] Q^{(\gamma-2) / 2}+\gamma(\gamma-2)\left(u_{i}-p_{i}\right)^{2} u_{i k}^{2} Q^{(\gamma-4) / 2},
\end{aligned}
$$

where $Q=1+\sum\left(u_{i}-p_{i}\right)^{2}+\left(u+p_{n+1}\right)^{2}, \tau_{k}=3$ if $k>1$ and $\tau_{1}=1$, and $p_{i, t}=d p_{i} / d t$. On the other hand, differentiating Eq. (1.6) gives

$$
\begin{aligned}
u_{k t} & =\sum_{i} u^{i i} u_{i i k}+g_{k}, \\
u_{k k t} & =\sum_{i} u^{i i} u_{i i k k}-\sum_{i, j} u^{i i} u^{j j} u_{i j k}^{2}+\log \operatorname{det} \nabla^{2} u+g_{k k},
\end{aligned}
$$

where $\left\{u^{i j}\right\}$ is the inverse matrix of $\left\{u_{i j}\right\}$. Hence at $(0, \bar{t})$ we have

$$
\begin{aligned}
0 \geqslant & \sum_{k} u^{k k} \varphi_{k k}-\varphi_{t} \\
\geqslant & \sum_{k} u^{k k} u_{k k 11}-u_{11 t}+u_{11} u^{k k} \\
& +\gamma\left\{\sum_{k} u_{k k}\left[1+\frac{(\gamma-2)\left(u_{k}-p_{k}\right)^{2}}{1+\sum\left(u_{i}-p_{i}\right)^{2}+\left(u+p_{n+1}\right)^{2}}\right]\right. \\
& +\left(u_{i}-p_{i}\right)\left(\sum_{k} u^{k k} u_{i k k}-u_{i t}\right)-n\left(u+p_{n+1}\right) \\
& \left.-\left(u+p_{n+1}\right)\left(u_{t}+p_{n+1, t}\right)+\left(u_{i}-p_{i}\right) p_{i, t}\right\} Q^{(\gamma-2) / 2} \\
\geqslant & u_{11} u^{k k}-\log \operatorname{det} \nabla^{2} u-g_{11}+\gamma\left[(\gamma-1) u_{k k}-\left(u_{i}-p_{i}\right) g_{i}\right. \\
& -n\left(u+p_{n+1}\right)-\left(u+p_{n+1}\right)\left(u_{t}+p_{n+1, t}\right) \\
& \left.+\left(u_{i}-p_{i}\right) p_{i, t}\right] Q^{(\gamma-2) / 2} .
\end{aligned}
$$

To proceed further let's assume $u_{11}>1$. By (2.2) we have $\left|u+p_{n+1}\right| \leqslant$ $2 D$ and $\left|u_{i}-p_{i}\right| \leqslant 2 D$. From the inequality above we therefore obtain, in view of (2.1),

$$
\begin{aligned}
& u_{k k}+u^{k k} \\
& \quad \leqslant C\left(1+\left|u_{t}\right|\right) Q^{(2-\gamma) / 2}+C\left(1+\left|u+p_{n+1}\right|\right)\left(1+\left|u_{t}\right|+\left|p_{n+1, t}\right|\right) \\
& \quad \leqslant C\left[1+D \log \left(u_{k k}+u^{k k}\right)+D \sup _{t \leqslant T} H_{t}(x, t)\right] .
\end{aligned}
$$


From Eq. (1.3),

$$
\sup _{t \leqslant T} H_{t}(x, t) \leqslant C+\log \left[\sup _{t<T}\left\{H_{\xi \xi}^{n}(x, t) ; x \in S^{n}, \xi \text { tangential to } S^{n}\right\}\right] .
$$

It follows

$$
u_{k k}+u^{k k} \leqslant C\left(1+D \log \left(u_{k k}+u^{k k}\right)\right) .
$$

Hence $u_{11} \leqslant C\left(1+D\left|\log ^{2} D\right|\right)$. This completes the proof of the lemma.

By combining Lemmas 2.2 and 2.4 we deduce the following important corollary.

LEMMA 2.5. - For any given $\gamma \in(1,2]$, there exists $\delta=\delta(\gamma)>0$ such that

$$
r(t) \geqslant \frac{\delta R^{2}(t)}{1+\sup _{\tau \leqslant t} R^{\gamma}(\tau)} .
$$

Next we give a positive lower bound for the principal radii of the curvature. In view of Lemma 2.4 and Eq. (1.3) it suffices to give a lower bound on $H_{t}$.

LEMMA 2.6. - There exists a constant $C$ depending only on $n, r_{0}, R_{0}$, $f$, and initial data such that

$$
\inf \left\{H_{t}(x, t):(x, t) \in S^{n} \times[0, T]\right\} \geqslant-C .
$$

Proof. - Let

$$
q(t)=\frac{1}{\left|S^{n}\right|} \int_{S^{n}} x H(x, t) d \sigma(x)
$$

be the Steiner point of $X(\cdot, t)$. Then there exists a positive $\delta$ which depends only on $n, r_{0}$, and $R_{0}$ so that $H(x, t)-q(t) \cdot x \geqslant 2 \delta$. Let us consider consider the function

$$
\Psi(x, t)=\frac{H_{t}(x, t)}{H(x, t)-x \cdot q(t)-\delta} .
$$

Suppose the (negative) infimum of $\Psi$ attains at $x=(0, \ldots, 0,-1)$ and $\bar{t}>0$. Let $u$ be the restriction of $H$ to $x_{n+1}=-1$ as before. Then

$$
\psi(y, t)=\frac{u_{t}(y, t)}{u(y, t)-q(t) \cdot(y,-1)-\delta \sqrt{1+|y|^{2}}}
$$


attains its negative minimum at $(0, \bar{t})$. Hence

$$
\begin{aligned}
& 0 \geqslant \psi_{t}=\frac{u_{t t}}{u+q_{n+1}(t)-\delta}-\frac{u_{t}\left(u_{t}+d q_{n+1} / d t\right)}{\left(u+q_{n+1}(t)-\delta\right)^{2}}, \\
& 0=\psi_{k}=\frac{u_{t k}}{u+q_{n+1}(t)-\delta}-\frac{u_{t}\left(u_{k}-q_{k}(t)\right)}{\left(u+q_{n+1}(t)-\delta\right)^{2}},
\end{aligned}
$$

and

$$
\begin{aligned}
0 \leqslant \psi_{k k}= & \frac{u_{t k k}}{u+q_{n+1}(t)-\delta}-\frac{u_{t} u_{k k}}{\left(u+q_{n+1}(t)-\delta\right)^{2}} \\
& +\frac{\delta u_{t}}{\left(u+q_{n+1}(t)-\delta\right)^{2}} .
\end{aligned}
$$

On the other hand, we differentiate (1.3) to get

$$
u_{t t}=u^{i j} u_{i j t}
$$

Rotate the axes so that $\left\{u^{i j}\right\}$ is diagonal at $(0, \bar{t})$. Then

$$
\begin{aligned}
0 & \leqslant \sum u^{k k} \psi_{k k}-\psi_{t} \\
& \leqslant \frac{\delta u_{t} \sum u^{k k}-n u_{t}+u_{t}\left(u_{t}+d q_{n+1} / d t\right)}{\left(u+q_{n+1}-\delta\right)^{2}} .
\end{aligned}
$$

Since $u_{t}$ is negative at $(0, \bar{t})$, it follows from Lemma 2.4 that

$$
\begin{aligned}
\sum u^{k k} & \leqslant \frac{n}{\delta}\left(1+\left|u_{t}\right|+\left|\frac{d q_{n+1}}{d t}\right|\right) \\
& \leqslant C \frac{n}{\delta}\left(1+\left|u_{t}\right|+R_{0}\right) \\
& \leqslant C \frac{n}{\delta}\left(1+\log \sum u^{k k}+R_{0}\right) .
\end{aligned}
$$

We therefore conclude $\sum u^{k k} \leqslant C \delta^{-2}\left(1+R_{0}\right)^{2}$. Hence

$$
\begin{aligned}
u_{t} & \geqslant-C-C \log \sum u^{k k} \\
& \geqslant-C\left(1+\log \left(1+R_{0}\right)-\log r_{0}\right)
\end{aligned}
$$

and the lemma follows.

Finally by comparing (1.3), (1.4) with the problem

$$
\frac{d \rho}{d t}=\log \rho^{n} M, \quad \rho(0)=\rho_{0}
$$


where $M=\max \left\{f(x): x \in S^{n}\right\}$ and $\rho_{0}$ is sufficiently large, we see that $H(x, t)$ is always bounded in any finite time interval. Furthermore, its gradient is also bounded by (1.1). It follows from the regularity property of fully nonlinear parabolic equations [11] that a $C^{4+\alpha, 2+\alpha / 2}$-estimate holds for $H$, provided $H_{0} \in C^{4+\alpha}\left(S^{n}\right), 0<\alpha<1$. By a continuity argument we arrive at

THEOREM 2.1. - The problem (1.3), (1.4) with $H_{0} \in C^{4+\alpha}\left(S^{n}\right)$ admits a unique $C^{4+\alpha, 2+\alpha / 2}$ solution in a maximal interval $\left[0, T^{*}\right), T^{*} \leqslant \infty$. Moreover, $\lim _{t \uparrow T^{*}} R(t)=0$ if $T^{*}$ is finite.

Notice that the last assertion follows from Lemma 2.5.

\section{PROOFS OF THEOREMS A AND B}

We first prove Theorem A. Let $m=\inf f$ and $M=\sup f$ on $S^{n}$. It is readily seen that if the initial hypersurface $X_{0}$ is a sphere of raduis $\rho_{0}>m^{-1 / n}$, the solution $X(\cdot, t)$ to the equation

$$
\frac{\partial X}{\partial t}=-\log \frac{K}{m} v, \quad X(\cdot, 0)=X_{0}
$$

remains to be spheres and the flow expands to infinity as $t \rightarrow \infty$. On the other hand, if $X_{0}$ is a sphere of radius less than $M^{-1 / n}$, the solution to

$$
\frac{\partial X}{\partial t}=-\log \frac{K}{M} v, \quad X(\cdot, 0)=X_{0}
$$

is a family of spheres which shrinks to a point in finite time. Henceforth by the comparison principle the solution $X(x, t)$ of (1.3), (1.4) will shrink to a point if $\theta$ is smalll enough, and will expand to infinity if $\theta>0$ is large. We put

$$
\theta_{*}=\sup \{\theta>0: X(\cdot, t) \text { shrinks to a point in finite time }\}
$$

and

$$
\theta^{*}=\inf \{\theta>0: X(\cdot, t) \text { expands to infinity as } t \rightarrow \infty\} .
$$

By the results in Section 2, it is easy to see that $X(\cdot, t)$ continuously depends on $\theta$. Hence by the comparison principle $\theta_{*} \leqslant \theta^{*}$.

By Lemma 2.5 we know that for any $\theta \in\left[\theta_{*}, \theta^{*}\right]$ the inner radii of $X(\cdot, t)$ have a uniform positive lower bound and the outer radii are 
unformly bound from above. Hence (1.3) is uniformly parabolic and we have $C^{4+\alpha, 2+\alpha / 2}$-bound on the solution in $S^{n} \times[0, \infty)$.

In the following we fix $\theta \in\left[\theta_{*}, \theta^{*}\right]$. Let $\xi \in \mathbf{R}^{n+1}$ be the point uniquely determined by

$$
\int_{S^{n}} \frac{x_{i}}{\mathrm{e}^{\xi \cdot x} f(x)} d \sigma(x)=0, \quad i=1, \ldots, n+1 .
$$

Write $\tilde{X}(x, t)=X(x, t)+\xi \cdot t$. So $\tilde{X}$ is $X$ translated in $\xi /|\xi|$ with speed $|\xi| . \tilde{X}$ satisfies

$$
\frac{\partial \tilde{X}}{\partial t}=-\log \frac{K}{f} v+\xi
$$

and the corresponding support function $\tilde{H}=H+\xi \cdot x t$ satisfies

$$
\tilde{H}_{t}=\log \operatorname{det}\left(\nabla_{\beta} \nabla_{\alpha} \tilde{H}+\tilde{H} \delta_{\alpha \beta}\right)+\log f \mathrm{e}^{\xi \cdot x} .
$$

The enclosed volumes of $\tilde{X}$ and $X$ are equal to

$$
V(t)=\frac{1}{n+1} \int \tilde{H} \operatorname{det}\left(\nabla_{\beta} \nabla_{\alpha} \tilde{H}+\tilde{H} \delta_{\alpha \beta}\right)
$$

and is uniformly bounded. On the other hand, by (3.1)

$$
\int \frac{\tilde{H}}{\mathrm{e}^{\xi \cdot x} f}=\int \frac{H-q(t) \cdot x}{f \mathrm{e}^{\xi \cdot x}}
$$

is also uniformly bounded for all $t$. Hence the functional $\tilde{J}(t)=$ $J(\tilde{H}(\cdot, t))$ is uniformly bounded. Moreover, from (1.5) it is nonincreasing. By the $C^{4+\alpha, 2+\alpha / 2}$-regularity of $\tilde{H}$ we also have that

$$
\left|\tilde{J}^{\prime}(t)\right| \leqslant C
$$

and

$$
\sup \frac{\left|\tilde{J}^{\prime}(t+\tau)-\tilde{J}^{\prime}(t)\right|}{\tau^{\alpha / 2}} \leqslant C .
$$

Therefore, we conclude that $\lim _{t \rightarrow \infty} \tilde{J}^{\prime}(t)=0$.

We claim that $\tilde{H}$ is bounded for all $t$. In fact, it is sufficient to show that $\int x \frac{\tilde{H}}{f \mathrm{e}^{\xi \cdot x}} d \sigma$ is bounded. For, assume $\tilde{H}$ is unbounded. Then we can 
find $\left\{t_{j}\right\}, t_{j} \rightarrow \infty$, such that $\tilde{X}\left(x, t_{j}\right) / d\left(t_{j}\right)$, where $d\left(t_{j}\right)$ is the distance from the origin to $\tilde{X}\left(\cdot, t_{j}\right)$, converges to a point on $S^{n}$. Without loss of generality we take this point to be $\boldsymbol{e}_{n+1}$. Then the characteristic functions of $A_{j}=\left\{x \in S^{n}: x_{n+1}>0, H\left(x, t_{j}\right)>0\right\}$ and $B_{j}=\left\{x \in S^{n}: x_{n+1}<0\right.$, $\left.H\left(x, t_{j}\right)<0\right\}$ converges pointwisely to the upper and lower hemispheres. We may also assume that $\tilde{H}\left(x, t_{j}\right) /\left(f \mathrm{e}^{\xi \cdot x} d\left(t_{j}\right)\right)$ converges uniformly to some function $g$ which is positive on the upper hemi-sphere $S^{+}$. Therefore, we have

$$
\begin{aligned}
\lim _{j \rightarrow \infty} \int \frac{x_{n+1} H\left(x, t_{j}\right)}{d\left(t_{j}\right) f \mathrm{e}^{\xi \cdot x}} & =\int \lim _{j \rightarrow \infty}\left[\mathcal{X}_{A_{j} \cup B_{j}} \frac{\left|x_{n+1} H\left(x, t_{j}\right)\right|}{d\left(t_{j}\right) f \mathrm{e}^{\xi \cdot x}}\right] \\
& \geqslant \int_{S^{+}} x_{n+1} g(x) \\
& >0 .
\end{aligned}
$$

Hence $\int \frac{x_{n+1} H\left(x, t_{j}\right)}{f \mathrm{e}^{\xi \cdot x}}$ can be arbitrarily large for large $t_{j}$.

Now we have, by (1.5),

$$
\tilde{J}(0)-\tilde{J}(\infty) \geqslant \int_{0}^{t}\left|\tilde{J}^{\prime}(t)\right| d t \geqslant \int_{0}^{t} \int_{S^{n}} \tilde{H}_{t}^{2} d \sigma d t .
$$

On the other hand, by the necessary condition for the Minkowski problem, we have

$$
\begin{aligned}
0 & =\int x \frac{1}{\tilde{K}} d \sigma=\int x \frac{1}{f \mathrm{e}^{\xi \cdot x}}\left(1+\tilde{H}_{t}+\mathrm{O}\left(\tilde{H}_{t}^{2}\right)\right) \\
& =\int x \frac{1}{f \mathrm{e}^{\xi \cdot x}}\left(\tilde{H}_{t}+\mathrm{O}\left(\tilde{H}_{t}^{2}\right)\right)
\end{aligned}
$$

as $\tilde{H}_{t}$ is uniformly small for large $t$. Therefore,

$$
\begin{aligned}
\left|\int_{0}^{t} \frac{d}{d t}\left(\int x \frac{\tilde{H}}{f \mathrm{e}^{\xi \cdot x}} d \sigma\right) d t\right| & \leqslant C \int_{0}^{t} \int_{S^{n}} \tilde{H}_{t}^{2} d \sigma d t \\
& \leqslant C(\tilde{J}(0)-\tilde{J}(\infty)) .
\end{aligned}
$$

Hence $\int x \frac{\tilde{H}}{f \mathrm{e}^{\xi \cdot x}}$ is uniformly bounded for all time. Consequently by the Blaschke selection theorem for any sequence $\left\{t_{j}\right\}, t_{j} \rightarrow \infty$, we can extract a subsequence $\left\{t_{j_{k}}\right\}$ such that $\left\{\tilde{H}\left(x, t_{j_{k}}\right)\right\}$ converges uniformly to some $H(x)$ on $S^{n}$. Clearly $H$ is a solution of $K=f \mathrm{e}^{\xi \cdot x}$. To show the convergence is actually uniform let's consider another limit $H^{\prime}$. Since the 
curvature of $H^{\prime}$ is also given by $f \mathrm{e}^{\xi \cdot x}, H$ and $H^{\prime}$ differ by a translation. Let $H-H^{\prime}=l \cdot x$ for some $l \in \mathbf{R}^{n+1}$. Since

$$
\left|\int_{s}^{t} \frac{d}{d t} \int x \frac{\tilde{H}}{f \mathrm{e}^{\xi \cdot x}} d \sigma d t\right| \leqslant C(\tilde{J}(t)-\tilde{J}(s)) \rightarrow 0
$$

as $t, s \rightarrow \infty$. So $l=0$ and $H=H^{\prime}$.

Finally let's show $\theta_{*}=\theta^{*}$. First we observe that by the comparison principle one must have $H_{*}=H^{*}$, where $H_{*}$ (respectively $H^{*}$ ) is the solution of $K=f \mathrm{e}^{\xi \cdot x}$ starting from $\theta_{*} H_{0}$ (respectively $\theta^{*} H_{0}$ ). However, consider the equation obtained by differentiating (1.3) and (1.4) in $\theta$ :

$$
\left\{\begin{array}{l}
\frac{\partial H^{\prime}}{\partial t}=A^{\alpha \beta}\left(\nabla_{\beta} \nabla_{\alpha} H^{\prime}+H^{\prime} \delta_{\alpha \beta}\right), \\
H^{\prime}(0)=H_{0}(x),
\end{array}\right.
$$

where $\left(A^{\alpha \beta}\right)$ is the inverse of $\left(\nabla_{\beta} \nabla_{\alpha} H+H \delta_{\alpha \beta}\right)$. By the maximum principle $H^{\prime}(x, t) \geqslant \min H_{0}>0$. Thus

$$
\begin{aligned}
0 & =H^{*}(\cdot)-H_{*}(\cdot) \\
& =\lim _{t \rightarrow \infty}\left(H_{\theta^{*}}(\cdot, t)-H_{\theta_{*}}(\cdot, t)\right) \\
& \geqslant\left(\min H_{0}\right)\left(\theta^{*}-\theta_{*}\right) \\
& >0 .
\end{aligned}
$$

So $\theta^{*}=\theta_{*}$. The proof of Theorem $\mathrm{A}$ is finished.

Proof of Theorem $B$. - It remains to show that the normalized hypersurface $X(\cdot, t) / r(t)$ converges to a unit sphere in case $\theta>\theta^{*}$. Let's denote the solution of (1.3), (1.4) by $H(\cdot, t)$ and its hypersurface by $X(\cdot, t)$. Since $X$ is expanding, we may simply assume that it contains the ball $B_{R_{1}}(0)$ where $R_{1}>1+m^{-1 / n}$ at $t=0$. On the other hand, we fix $R_{2}$ so large that $X(\cdot, 0)$ is contained in $B_{R_{2}}(0)$.

For $i=1,2$, let $X_{i}(\cdot, t)$ be the solution of (1.3), (1.4) where $f$ is replaced by $m$ and $M$ respectively and $X_{i}(\cdot, 0)=\partial B_{R_{i}}$. Clearly $X_{i}(\cdot, t)$ are spheres whose radii $R_{i}(t)$ satisfy

$$
\begin{aligned}
C^{-1}(1+t) \log (1+t) & \leqslant R_{1}(t) \\
& \leqslant R_{2}(t) \\
& \leqslant C\left[1+(1+t) \log ^{2}(1+t)\right]
\end{aligned}
$$

for some $C>0$. Hence 


$$
\begin{aligned}
\frac{d}{d t}\left(R_{2}(t)-R_{1}(t)\right) & \leqslant n \log \frac{R_{2}(t)}{R_{1}(t)}+C \\
& \leqslant \log \log (1+t)+C
\end{aligned}
$$

and so

$$
R_{2}(t)-R_{1}(t) \leqslant C[1+t \log \log (1+t)] .
$$

Consequently $\lim _{t \rightarrow 0} \frac{R_{2}(t)-R_{1}(t)}{R_{1}(t)}=0$. By the comparision principle $X(\cdot, t)$ is pinched between $X_{2}(\cdot, t)$ and $X_{1}(\cdot, t)$. So $X(\cdot, t) / r(t)$ must tend to the unit sphere uniformly.

\section{ACKNOWLEDGEMENT}

The first auther was partially supported by an Earmarked Grant for Research. The second auther was supported by NNSF of China.

\section{REFERENCES}

[1] Andrews B., Contraction of convex hypersurfaces by their affine normal, J. Differential Geom. 43 (1996) 207-229.

[2] Andrews B., Evolving convex curves, Calc. Var. PDE 1 (1998) 315-371.

[3] Cheng S.Y., Yau S.T., On the regularity of the solution of the $n$-dimensional Minkowski problem, Comm. Pure Appl. Math. 29 (1976) 495-516.

[4] Chou K. (Tso, K.), Deforming a hypersurface by its Gauss-Kronecker curvature, Comm. Pure Appl. Math 38 (1985) 867-882.

[5] Chou K. (Tso, K.), Convex hypersurfaces with prescribed Gauss-Kronecker curvature, J. Differential Geom. 34 (1991) 389-410.

[6] Chou K., Zhu X., Anisotropic curvature flows for plane curves, Duke Math. J. 97 (1999) 579-619.

[7] Chow B., Deforming convex hypersurfaces by the $n$-th root of the Gaussian curvature, J. Differential Geom. 22 (1985) 117-138.

[8] Firey W., Shapes of worn stones, Mathematica 21 (1974) 1-11.

[9] Gage M.E., Li Y., Evolving plane curves by curvature in relative geometries II, Duke Math. J. 75 (1994) 79-98.

[10] Gerhardt C., Flow of non convex hypersurfaces into spheres, J. Differential Geom. 32 (1990) 299-314.

[11] Krylov N.V., Nonlinear Elliptic and Parabolic Equations of the Second Order, D. Reidel, 1987.

[12] Lewy H., On differential geometry in the large, I (Minkowski's problem), Trans. Amer. Math. Soc. 43 (1938) 258-270.

[13] Minkowski H., Allgemeine Lehrsätze über die konvexen Polyeder, Nachr. Ges. Wiss. Göttingen (1897) 198-219.

[14] Minkowski H., Volumen and Oberfläche, Math. Ann. 57 (1903) 447-495. 
[15] Miranda C., Su un problema di Minkowski, Rend. Sem. Mat. Roma 3 (1939) 96108.

[16] Nirenberg L., The Weyl and Minkowski problems in differential geometry in the large, Comm. Pure Appl. Math. 6 (1953) 337-394.

[17] Pogorelov A.V., The Multidimensional Minkowski Problem, J. Wiley, New York, 1978.

[18] Urbas J.I.E., On the expansion of convex hypersurfaces by symmetric functions of their principal radii of curvature, J. Differential Geom. 33 (1991) 91-125. 